\title{
Intact fibroblast growth factor 23 tested by a fully automated assay: clinical correlates and prognostic value in chronic systolic heart failure
}

\section{Giuseppe Vergaro ( $\nabla$ vergaro@ftgm.it ) \\ Fondazione Toscana Gabriele Monasterio \\ Annamaria Del Franco}

Scuola Superiore Sant'Anna

Francesco Gentile

Fondazione Toscana Gabriele Monasterio

Vincenzo Castiglione

Fondazione Toscana Gabriele Monasterio

Alberto Aimo

Scuola Superiore Sant'Anna

\section{Federica Saponaro}

University of Pisa

\section{Silvia Masotti}

Fondazione Toscana Gabriele Monasterio

\section{Concetta Prontera}

Fondazione Toscana Gabriele Monasterio

\section{Niccolò Fusari}

Fondazione Toscana Gabriele Monasterio

Michele Emdin

Scuola Superiore Sant'Anna

Claudio Passino

Scuola Superiore Sant'Anna

\section{Research Article}

Keywords: Fibroblast growth factor, Heart failure, Calcium-phosphate metabolism, Prognosis

Posted Date: February 7th, 2022

DOI: https://doi.org/10.21203/rs.3.rs-1256999/v1 
License: (c) (i) This work is licensed under a Creative Commons Attribution 4.0 International License. Read Full License 


\section{Abstract}

Background Fibroblast growth factor-23 (FGF23) has been associated to left ventricular (LV) hypertrophy and heart failure (HF) severity. We aimed to investigate clinical correlates and prognostic value of intact FGF23 (iFGF23) in HF patients.

Methods Patients with systolic HF (left ventricular ejection fraction - LVEF $<50 \%$ ) were prospectively enrolled. iFGF23 was tested with a fully automated immuno-chemiluminescent assay. Patients were divided into iFGF23 tertiles and followed-up for all-cause death, cardiac death, and the composite of allcause death and HF hospitalizations.

Results We enrolled 150 patients ( $82 \%$ males; median age 65 years). First, second, and third iFGF23 tertiles were $<35.2 \mathrm{pg} / \mathrm{mL}, 35.2-50.9 \mathrm{pg} / \mathrm{mL}$, and $>50.9 \mathrm{pg} / \mathrm{mL}$. Patients in the upper iFGF23 tertile had lower LVEF $(p=0.014)$, higher NT-proBNP $(p=0.001)$, and lower peak $\mathrm{VO}_{2}(p<0.001)$. Phosphate, estimated glomerular filtration rate (eGFR), and LV mass index were independently associated to iFGF23 (all $p<0.05)$. Patients in the upper tertile were at higher risk of all-cause death $(p=0.001)$, cardiac death $(p=0.001)$, and of the composite of all-cause death and HF hospitalizations $(p=0.003)$. iFGF-23 was an independent predictor of all the end-points after adjustment for age, LVEF, eGFR, and NT-proBNP.

Conclusions Circulating iFGF23 is associated with renal dysfunction, disease severity and outcome in systolic HF.

\section{Introduction}

Fibroblast growth factor 23 (FGF23) is a peptide hormone mainly synthetized in the bone, regulating phosphate balance by blocking renal tubular phosphate reabsorption and inhibiting the synthesis of 1,25dihydroxyvitamin D [1]. Moreover, FGF23 has been shown to have pleiotropic effects beyond the regulation of calcium-phosphate homeostasis [2]. FGF23 receptors are indeed expressed in the heart, and several experimental and clinical studies support a role of FGF23 in the development of left ventricular (LV) hypertrophy, fibrosis, and dysfunction [3-6] in different settings, including chronic kidney disease, mostly through an interplay with the renin-angiotensin-aldosterone system (RAAS) [3]. The combination of increased FGF23 and low Klotho - a FGF23 cofactor - levels is associated with a higher risk of cardiovascular death or heart failure (HF) hospitalization in subjects with stable ischemic heart disease. Similarly, the elevation of FGF23 is associated with a significantly increased risk of incident HF in hypertensive populations $[7,8]$.

Over the past years, FGF23 has emerged as a possible HF biomarker [9]. For example, in the BIOlogy Study to TAilored Treatment in Chronic HF (BIOSTAT-CHF) study, the elevation of FGF23 identified a subset of HF patients with more severe disease and is associated to impaired up-titration of angiotensinconverting enzyme inhibitors (ACEi) and angiotensin receptor blockers (ARBs) [10]. While previous studies had shown that FGF23 is independently associated with cardiovascular mortality and with the composite endpoint of death or heart transplantation in patients with systolic HF [9,11], a recent post-hoc analysis of 
the Trial of Intensified vs Standard Medical Therapy in Elderly Patients With Congestive Heart Failure (TIME-CHF) has questioned the predictive power of FGF23 [12].

Furthermore, all the studies published so far have reported the clinical value of FGF23 tested with enzyme-linked immunosorbent assays directed to either C-terminal FGF23 (cFGF23) or to the intact, biologically active hormone (iFGF23). A novel, fully automated FGF23 assay measuring selectively iFGF23 has become available [13], but the clinical significance of iFGF23 in patients with HF remains to be elucidated.

We aimed to assess correlates and prognostic value of iFGF23, tested with an automated assay, in a fully characterized cohort of patients with systolic HF.

\section{Materials And Methods}

\section{Study_population}

We enrolled 150 consecutive patients with systolic HF (left ventricular ejection fraction - LVEF $<50 \%$ ), in a tertiary referral centre for HF (Fondazione Toscana Gabriele Monasterio, Pisa, Italy), between June 2015 and December 2016. All patients were on stable guideline-recommended HF therapy since at least 2 months. Diagnosis of chronic HF was made according to contemporary European Society of Cardiology guidelines [14]. Exclusion criteria were acute coronary syndrome or HF decompensation, coronary artery revascularization or cardiac resynchronization therapy within 3 months before enrolment. Patients underwent resting 12-lead electrocardiogram, biohumoral characterization, echocardiography and cardiopulmonary exercise testing (CPET). Standard 2D echocardiography was performed to evaluate LV geometry, and indices of systolic and diastolic function, according to standard procedures [15].

The study protocol conforms to the 1975 Declaration of Helsinki and was approved by the Ethical Committee of the Outer North West Area, Tuscany (Italy). Written informed consent was obtained from each patient.

\section{Biohumoral assays}

Blood samples were drawn after an overnight fasting period and a 20-minute supine rest [16], and then stored at $-80^{\circ} \mathrm{C}$ until assays were performed. Plasma renin activity (PRA) and aldosterone were measured using a radioimmunoassay method (DiaSorin S.r.l., Saluggia, Italy) [17,18]. Plasma epinephrine and norepinephrine were evaluated by means of high-performance liquid chromatography technique using the electrochemical detector CLC 100 (Chromsystems GmbH, München, Germany) [19]. N-terminal fraction of pro-B-type natriuretic peptide (NT-proBNP) was measured with an electrochemiluminescence immunoassay monoclonal method using the Cobas e411 platform (Roche Diagnostics Italia, Monza, Italy) [20]. 25 hydroxy-vitamin D3 (250HD) was measured by isotope dilution (HPLC-MS/MS), by using the MSMS vitamin D Kit from PerkinElmer (Waltham, MA, USA). Agilent 1290 Infinity UHPLC system was 
used (Santa Clara, CA, USA), including autosampler, binarypump, and column oven, coupled to an AB Sciex API 4000 triple quadrupole mass spectrometer (Concord, ON, Canada), equipped with an APCI source. Chromatography was performed by a PerkinElmer Brownlee Supra C18 $3 \mu \mathrm{m}, 50 \times 2.1 \mathrm{~mm} \mathrm{HPLC}$ column, protected by a PerkinElmer Brownlee Supra C18 Guard Column. Plasma parathyroid hormone (PTH) was measured by the third-generation assay 1-84 PTH assay, chemiluminescent immunoassay (DiaSorin, Saluggia, Italy). Finally, for FGF23 evaluation, an automated iFGF23 immunochemiluminescent sandwich assay (DiaSorin, Saluggia, Italy; LoD $<10 \mathrm{ng} / \mathrm{L}$, intra-assay CV $<3 \%$, interassay CV $<7 \%$ ) was employed [13].

All these assays were performed according to manufacturer instructions. Estimated glomerular filtration rate (eGFR) was calculated with the Chronic Kidney Disease Epidemiology Collaboration (CKD-EPI) formula.

\section{Survival analysis and endpoints}

Follow-up was performed every 3 to 6 months unless otherwise clinically required. Data were collected by two independent interviewers directly from patients, relatives, or general practitioners, while the time and the precise cause of death were retrieved from death certificates, post-mortem reports, and general practitioners. The primary endpoint was all-cause death, while the secondary endpoints were cardiac death (including sudden cardiac death and death due to HF progression or acute myocardial infarction) and the composite of all-cause death and HF hospitalizations. Patients were censored after the last medical contact.

\section{Statistical analysis}

After evaluation for normality by Kolmogorov-Smirnov test, normally distributed variables were reported as mean \pm standard deviation while skewed variables as median and interquartile interval (IQI). Categorical data were reported as frequencies. According to variable distribution, ANOVA or KruskalWallis test were used for multiple comparisons among groups, with post hoc Dunn-Bonferroni correction for pairwise comparisons. $\chi 2$ or Fisher test were adopted for qualitative variables. Linear regression models were employed to evaluate correlates of iFGF23 levels, including clinical parameters (age, gender, body mass index - BMI, New York Heart Association - NYHA - class), laboratory (eGFR, haemoglobin, phosphate, PTH, 250HD, calcium, aldosterone, PRA and catecholamine levels), and echocardiography parameters (LVEF, pulmonary artery systolic pressure - PAPs, LV mass index - LVMI, E/e' ratio). All variables with $p<0.05$ at univariate analysis were included in a multivariate regression model.

For survival analysis, the first iFGF23 tertile was considered as reference category. Kaplan-Meier method and Log-Rank statistics (Mantel-Cox) were used to estimate all-cause mortality and cardiac death, according to iFGF23 tertiles. A multivariable Cox proportional hazard model was built to manage possible confounders, selected a priori and including age, eGFR, LVEF and NT-proBNP. Net reclassification 
improvement (NRI, with risk categories set at $<10 \%, 10-30 \%$ and $>30 \%$ ) and the integrated discrimination improvement (IDI) were calculated to assess reclassification.

Statistical analysis was performed using SPSS (IBM Statistics, version 26.0, 2019). A two-tailed $p$ value $\leq 0.05$ was considered significant.

\section{Results}

\section{Baseline characteristics and comparison among iFGF23 tertiles}

We enrolled 150 consecutive patients with systolic HF (median LVEF 32\%, IQI 27-38\%). Median age was 65 years (54-74 years), $82 \%$ were males, and $72 \%$ were in NYHA class $\mathrm{H}-\mathrm{II}$. Renal function was mainly preserved (eGFR $73 \pm 24 \mathrm{~mL} / \mathrm{min} / 1.73 \mathrm{~m} 2$ ). Further, NT-proBNP plasma levels (953 ng/L, 354-2024 ng/L) demonstrated a moderate extent of neurohormonal activation, despite the use of an optimal medical therapy ( $87 \%$ of population treated with $\beta$-blockers, $61 \%$ with ACEi, $31 \%$ with ARBs and $61 \%$ with mineralocorticoid receptor antagonists - MRAs).

Median iFGF23 was $41.8 \mathrm{pg} / \mathrm{mL}(30.6-55.6 \mathrm{pg} / \mathrm{mL})$ and the first, second, and third tertile were $<35.2$ $\mathrm{pg} / \mathrm{mL}, 35.2-50.9 \mathrm{pg} / \mathrm{mL}$, and $>50.9 \mathrm{pg} / \mathrm{mL}$, respectively. Baseline characteristics of the overall population and differences among iFGF23 tertiles are shown in detail in Table 1 and Figure 1. Compared to patients in the first tertile, patients in the third tertile of iFGF23 showed lower maximal oxygen consumption at CPET and lower LVEF at echocardiography. In addition, compared to the first and second tertile of iFGF23, patients in the upper tertile showed higher levels of NT-proBNP and lower eGFR. Patients in the upper tertile of iFGF23 showed higher PTH than patients with lower levels of iFGF23, while no significant difference could be found for phosphate or 250HD.

\section{Table 1}

Baseline characteristics of the overall population and by tertiles of intact fibroblast growth factor 23 (iFGF23). 


\begin{tabular}{|c|c|c|c|c|c|}
\hline & $\begin{array}{l}\text { Overall } \\
(n=150)\end{array}$ & $\begin{array}{l}\text { iFGF23 } \\
<35.2 \mathrm{pg} / \mathrm{mL} \\
(\mathrm{n}=50)\end{array}$ & $\begin{array}{l}\text { iFGF23 } \\
35.2-50.9 \\
\mathrm{pg} / \mathrm{mL} \\
(\mathrm{n}=50)\end{array}$ & $\begin{array}{l}\text { iFGF23 } \\
>50.9 \mathrm{pg} / \mathrm{mL} \\
(n=50)\end{array}$ & $\begin{array}{l}p \\
\text { value } \\
\text { (trend) }\end{array}$ \\
\hline Age, years & $65(54-74)$ & 63 (54-72) & $68(54-76)$ & $67(57-75)$ & 0.325 \\
\hline Male, n (\%) & $123(82)$ & $39(78)$ & $43(86)$ & $41(82)$ & 0.501 \\
\hline $\mathrm{BMI}, \mathrm{Kg} / \mathrm{m}^{2}$ & $\begin{array}{l}26.2(23.9- \\
29.8)\end{array}$ & $\begin{array}{l}26.0(23.6- \\
29.1)\end{array}$ & $\begin{array}{l}26.2(24.5- \\
26.7)\end{array}$ & $\begin{array}{l}26.8(23.0- \\
31.5)\end{array}$ & 0.844 \\
\hline NYHA class & & & & & 0.152 \\
\hline I & $39(26)$ & $17(34)$ & $13(26)$ & $9(18)$ & \\
\hline II-III & $111(74)$ & $33(66)$ & 37 (74) & $41(82)$ & \\
\hline $\begin{array}{l}\text { Atrial fibrillation, } \\
n(\%)\end{array}$ & $21(28)$ & $5(10)$ & $12(24)$ & $4(8)$ & 0.036 \\
\hline $\begin{array}{l}\text { Peak } \mathrm{VO}_{2} \\
\mathrm{~mL} /(\mathrm{kg} \cdot \mathrm{min})\end{array}$ & $\begin{array}{l}13.6(10.8- \\
18.0)\end{array}$ & $\begin{array}{l}17.1(12.4- \\
21.3)\end{array}$ & $\begin{array}{l}14.2(11.1- \\
18.4)\end{array}$ & $\begin{array}{l}12.3(10.0- \\
13.9)^{\star}\end{array}$ & $<0.001$ \\
\hline LVEF, \% & $32(27-38)$ & $35(30-39)$ & $35(28-40)$ & $30(25-38)^{*}+$ & 0.014 \\
\hline LVMI, g/m² & $\begin{array}{l}129(112- \\
157)\end{array}$ & $\begin{array}{l}123(109- \\
143)\end{array}$ & $132(120-164)$ & $137(115-178)$ & 0.103 \\
\hline PAPs, mmHg & $35(29-40)$ & $32(29-38)$ & $35(29-40)$ & $36(30-43)$ & 0.102 \\
\hline$E / e^{\prime}$ & $12(8-16)$ & $9(7-12)$ & $13(10-16)$ & $13(7-18)$ & 0.089 \\
\hline Haemoglobin $(\mathrm{g} / \mathrm{dL})$ & $13.6(1.5)$ & $13.9(1.1)$ & $13.4(1.6)$ & $13.7(1.7)$ & 0.335 \\
\hline $\begin{array}{l}\text { eGFR, } \mathrm{mL} / \mathrm{min} / 1.73 \\
\mathrm{~m}^{2}\end{array}$ & $73 \pm 24$ & $86 \pm 20$ & $75 \pm 20 *$ & $59 \pm 23^{*}+$ & $<0.001$ \\
\hline PTH, pg/mL & $\begin{array}{l}19.6(14.3- \\
27.9)\end{array}$ & $\begin{array}{l}18.4(12.3- \\
25.4)\end{array}$ & $\begin{array}{l}17.8(13.2- \\
25.1)\end{array}$ & $\begin{array}{l}22.1(16.7- \\
35.0)^{*+}\end{array}$ & 0.003 \\
\hline Phosphate, mg/dL & $3.5(3.0-4.1)$ & $3.4(3.0-3.9)$ & $3.5(3.0-4.0)$ & $3.7(3.1-4.4)$ & 0.267 \\
\hline Calcium, mg/dL & $\begin{array}{l}9.00(8.75- \\
9.20)\end{array}$ & $\begin{array}{l}9.00(8.70- \\
9.23)\end{array}$ & $\begin{array}{l}8.90(8.70- \\
9.10)\end{array}$ & $\begin{array}{l}9.10(9.00- \\
9.30) \dagger\end{array}$ & 0.004 \\
\hline $\begin{array}{l}\text { 25-hydroxyvitamin } \\
\text { D3, ng/mL }\end{array}$ & $\begin{array}{l}16.26(9.40- \\
24.49)\end{array}$ & $\begin{array}{l}15.19(7.60- \\
21.50)\end{array}$ & $\begin{array}{l}20.53(13.63- \\
25.97)\end{array}$ & $\begin{array}{l}15.83(9.09- \\
25.44)\end{array}$ & 0.078 \\
\hline NT-proBNP, ng/L & $\begin{array}{l}953(354- \\
2024)\end{array}$ & $\begin{array}{l}686(219- \\
1349)\end{array}$ & $\begin{array}{l}822(376- \\
1567)\end{array}$ & $\begin{array}{l}1680(635- \\
3285)^{\star+}\end{array}$ & 0.001 \\
\hline PRA, ng/mL/h & $\begin{array}{l}1.31(0.30- \\
3.94)\end{array}$ & $\begin{array}{l}0.71(0.20- \\
2.40)\end{array}$ & $\begin{array}{l}1.13(0.21- \\
3.15)\end{array}$ & $\begin{array}{l}2.37(0.75- \\
7.44)^{\star}\end{array}$ & 0.003 \\
\hline
\end{tabular}




\begin{tabular}{llllll} 
Aldosterone, ng/dL & $\begin{array}{l}137.8(89.4- \\
209.4)\end{array}$ & $\begin{array}{l}137.8(98.9- \\
190.7)\end{array}$ & $\begin{array}{l}105.5(71.7- \\
179.6)\end{array}$ & $\begin{array}{l}168.8(91.4- \\
322.2) \dagger\end{array}$ & 0.027 \\
\hline Epinephrine, ng/L & $22(10-52)$ & $18(10-45)$ & $18(10-51)$ & $27(10-60)$ & 0.575 \\
\hline Norepinephrine, ng/L & $\begin{array}{l}404(283- \\
595)\end{array}$ & $\begin{array}{l}388(242- \\
621)\end{array}$ & $377(241-523)$ & $510(316-628)$ & 0.126 \\
\hline ACEi, n (\%) & $92(61)$ & $33(66)$ & $30(60)$ & $29(58)$ & 0.557 \\
\hline ARBs, n (\%) & $46(31)$ & $17(34)$ & $12(24)$ & $17(34)$ & 0.493 \\
\hline Beta blockers, n (\%) & $130(87)$ & $43(86)$ & $44(88)$ & $43(86)$ & 0.708 \\
\hline MRAs, n(\%) & $92(61)$ & $30(60)$ & $28(56)$ & $34(68)$ & 0.616 \\
\hline Diuretics, n (\%) & $104(69)$ & $31(62)$ & $35(70)$ & $38(76)$ & 0.451
\end{tabular}

ACEi, angiotensin-converting-enzyme inhibitors; ARBs, Angiotensin II receptor blockers; BMI, body mass index; eGFR, estimated Glomerular Filtration Rate; LVEF, left ventricular ejection fraction; LVMI, left ventricular mass index; MRAs: mineralocorticoid receptor antagonists; NT-proBNP, N-terminal pro B-type natriuretic peptide; PAPs, pulmonary artery systolic pressure; PRA, plasma renin activity; PTH, parathormone.

$* \mathrm{P}<0.05$ vs. first tertile of FGF23, $+\mathrm{P}<0.05$ vs. second tertile of FGF23.

\section{Clinical and biohumoral correlates of iFGF23}

Univariate predictors of iFGF23 are listed in Table 2. When all univariate predictors were pooled in a multivariable analysis, only eGFR (standardized $\beta=-0.422, p<0.001$ ), phosphate (standardized $\beta=0.179$, $p=0.024$ ) and LVMI (standardized $\beta=0.188, p=0.033$ ) were independently correlated to iFGF23 levels.

\section{Table 2}

Clinical correlates of intact fibroblast growth factor 23 (iFGF23) 


\section{Non- $\quad 95 \%$ confidence interval Standardized Beta \\ stardardized B for B

\begin{tabular}{lllll}
\hline LVEF & 0.007 & -0.003 to 0.018 & 0.137 & 0.171 \\
\hline NYHA Class & -0.044 & -0.148 to 0.060 & -0.073 & 0.404 \\
\hline PASP & 0.005 & $-0-002$ to 0.012 & 0.128 & 0.147 \\
\hline LVMI & 0.002 & 0.000 to 0.004 & 0.188 & 0.033 \\
\hline NT-proBNP & 0.000 & 0.000 to 0.000 & 0.126 & 0.248 \\
\hline eGFR & -0.008 & -0.011 to -0.004 & -0.422 & $<0.001$ \\
\hline PTH & -0.001 & -0.007 to 0.004 & -0.039 & 0.672 \\
\hline Phosphate & 0.090 & 0.012 to 0.167 & 0.179 & 0.024 \\
\hline PRA & 0.035 & -0.021 to 0.090 & 0.113 & 0.216 \\
\hline Aldosterone & -0.017 & -0.121 to 0.086 & -0.030 & 0.739 \\
\hline Norepinephrine & 0.015 & -0.105 to 0.135 & 0.019 & 0.808
\end{tabular}

Univariate predictors of iFGF23 concentration have been pooled into a multivariable model. Only LVMI, eGFR, and phosphate were independently associated to iFGF23. eGFR, estimated Glomerular Filtration Rate; LVEF, left ventricular ejection fraction; LVMI, left ventricular mass index; NT-proBNP, N-terminal pro Btype natriuretic peptide; PASP, pulmonary artery systolic pressure; PRA, plasma renin activity; PTH, parathormone.

\section{Prognostic impact of iFGF23 on mortality.}

During a median follow-up of 70 months (range 2-135), 25 (17\%) patients died, 14 of them due to cardiac causes; 24 HF hospitalizations were recorded. Decedents had higher iFGF23 than survivors (56.3 [44.488.3] pg/mL vs 39.6 [29-4-52.7] pg/mL, $p<0.001$ ). In unadjusted Cox proportional hazard model, iFGF23 levels significantly predicted all-cause death ( $\mathrm{HR}=7.62,95 \% \mathrm{Cl}$ [3.17-18.35]; $p<0.001)$, cardiac death $(\mathrm{HR}=7.43,95 \% \mathrm{Cl}[2.29-24.06] ; \mathrm{p}=0.001)$, and the composite of all-cause death and HF hospitalization $(H R=5.39,95 \% \mathrm{Cl}[2.52-11.52] ; p<0.001)$. After multivariable adjustment including age, eGFR, LVEF and NT-proBNP, the association between iFGF23 and outcome was maintained (all-cause death: $\mathrm{HR}=4.27$, 95\% Cl [1.43-12.79], $p=0.009$; cardiac death: $\mathrm{HR}=3.29,95 \% \mathrm{Cl}$ [0.76-14.29], $\mathrm{p}=0.011$; all-cause death and HF hospitalization: $\mathrm{HR}=2.89,95 \% \mathrm{Cl}[1.14-7.38], \mathrm{p}=0.026)$. FGF23 also improved reclassification over NTproBNP for the composite end-point (NRI=0.335, 95\% Cl [-0.050- 0.720], $p=0.088$; IDI=0.068, 95\% Cl [0.0050.131], $p=0.034)$. Furthermore, patients in the upper tertile of iFGF23 were at higher risk of 5-year all-cause (Log-rank 13.62, p=0.001) (Figure 2a), cardiac death (Log-rank 14.31, p=0.001) (Figure 2b) and of the 
composite of all-cause death and HF hospitalization (Log-rank 11.85, $\mathrm{p}=0.003$ ) (Figure 2c) compared to patients in the lower and middle tertile.

\section{Discussion}

In our fully characterized cohort of patients with stable HF, higher iFGF23, tested with a fully automated assay, was associated with clinical severity of the disease, as expressed by lower LV systolic function and higher circulating levels of NT-proBNP, and impaired functional capacity, demonstrated by lower peak $\mathrm{VO}_{2}$ at CPET. Moreover, iFGF23 elevation identified patients at higher risk for all-cause and cardiac mortality, independently of NT-proBNP and other established markers, including renal function (Figure 3).

\section{Correlates of iFGF23}

FGF23 plays a key role in regulating phosphate homeostasis. Elevated FGF23 levels increase fractional phosphate excretion at kidney level and inhibit PTH synthesis and secretion by parathyroid cells [21]. On the other hand,phosphate itself is a major regulator of FGF23:dietary phosphate loading increases circulating FGF23 levels, whereas phosphate depletion decreases FGF23 expression [22]. The evidence of the independent association between iFGF23 and phosphate in our population is therefore supported by pathophysiological mechanisms of FGF23 regulation.

Renal function, assessed byeGFR, emerged as another independent predictor of iFGF23 in our study. The inverse correlation between eGFR and iFGF23 is likely due to both direct and indirect mechanisms, consisting in the reduced renal excretion of iFGF23 and increased phosphate levels, respectively, following decreasing renal function.

There is experimental evidence that FGF23 may enhance RAAS activity [23], although more recent clinical data are controversial [7]. An analysis from the Multi-Ethnic Study of Atherosclerosis (MESA) study could not demonstrate a relationship between iFGF23 and either circulating aldosterone or PRA in hypertensive patients [7]. Conversely, data from a large population with new onset or worsening HF have shown that aldosterone levels were independently associated with higher FGF23 [10]. This is the first study addressing the possible association between biohumoral indices of RAAS activation and iFGF23 in the setting of chronic HF. In our population, both aldosterone and PRA were associated to iFGF23 circulating levels at univariate analysis, but the association was lost after multivariate adjustment.

Stimulation of RAAS activity has been postulated as one of the mechanisms underlying the association between FGF23 and LV hypertrophy [5]. Available data also suggest that activated RAAS induces myocardial expression of FGF23 [24], that could in turn promote fibrosis-related pathways in fibroblasts and consequently cardiac remodelling and dysfunction [25]. Experimental studies show how transgenic mice with overexpression of a constitutively active FGF receptor-1 develop LV hypertrophy [26]. Further, FGF23 blockade reverses the hypertrophic growth of isolated myocytes in vitro and established LV hypertrophy in vivo[27].We report here that LVMI is independently associated with iFGF23 in a cohort of 
stable systolic HF patients. These findings could support the hypothesis that FGF23 is not only a biomarker of increased risk but acts as a direct endocrine and paracrine player in the pathophysiology of HF.

\section{iFGF23 and outcome in stable HF patients}

Our findings that iFGF23 is an independent predictor of outcome in patients with $\mathrm{HF}$ is in line with previous evidence reporting FGF23 as a correlate of HF-related outcome,in patient with or without kidney disease [7], as well as a predictor of cardiovascular death in a small HF cohort [11]. Recently, data from 382 patients enrolled in the TIME-CHF (Trial of Intensified vs. Standard Medical Therapy in Elderly Patients With Congestive Heart Failure) cohort have questioned the role of FGF23 in risk stratification [12]. Still, this analysis included patients with more severe disease (no patient in NYHA class I vs. $26 \%$ in our population), and bothcFGF23 and iFGF23 were tested with commercially available ELISA assays. We used rather afully automated immunoassay for iFGF23 that is approved for clinical use and shows excellent analytical characteristics, allowing standard results and references. In addition, this assay measures the intact form of (iFGF23), showing a stronger biological activity than cFGF23 [28]. Furthermore, all blood samples were performed at the same time of the day, to avoid potential circadian variations of FGF23 levels between patients, and testing included a full characterization of RAAS activation as well as of phosphate and calcium metabolism.

\section{Significance}

FGF23 is increasingly recognized as a marker and a mediator of disease progression in HF. Our findings support the role of iFGF23 as a player in cardiac remodelling following renal dysfunction and a tool for risk stratification in HF patients. Characterization of the clinical significance of iFGF23 is of great relevance given the availability of different pharmacological approaches for FGF23 blockade. These include inhibitors of gastrointestinal sodium/hydrogen exchanger isoform 3 such as tenapanor [29], and cinecalcet, a calcimimetic agent that has been shown to reduce both serum FGF23 levels and rates of cardiovascular death and major cardiovascular events in haemodialysis patients [30]. Finally, burosumab, a monoclonal antibody targeting FGF23, has been recently tested in patients with X-linked hypophosphatemia [31].

\section{Limitations}

Serial measurements of iFGF23 have not been performed in the present study, so changes in iFGF23 during the follow-up and their influence on patient outcome can not be assessed. Moreover, we report data from a single tertiary centre, possibly limiting the generalizability of our findings. Considering the limited number of enrolled patients, a re-classification of FGF23 tertiles based on LVEF could not be performed. 


\section{Conclusion}

Circulating iFGF23, assessed with a novel fully automated assay, is associated with renal dysfunction, disease severity and outcome in a population of stable systolic HF patients. The relevance of iFGF23 as a marker in this clinical setting is supported by its biological role in prohypertrophic and profibrotic pathways, mostly activated both in renal and cardiac failure and sustaining cardiac remodelling, all this suggesting FGF23 as a possible therapeutic target in HF patients.

\section{Abbreviations}

25OHD, 25 hydroxy-vitamin D3

ACEi, angiotensin-converting enzyme inhibitors;

ARBs, angiotensin receptor blockers;

BMI, body mass index;

cFGF23, C-terminal fraction of FGF23;

eGFR, estimated glomerular filtration rate;

FGF23, fibroblast growth factor-23;

$H F$, heart failure;

iFGF23, intact FGF23;

$L V$, left ventricular;

LVEF, left ventricular ejection fraction;

LVMI, left ventricular mass index;

MRAs, mineralocorticoid receptor antagonists;

NT-proBNP, N-terminal fraction of pro-B-type natriuretic peptide;

NYHA, New York Heart Association;

PASP, pulmonary artery systolic pressure;

PRA, plasma renin activity;

PTH, parathyroid hormone; 


\section{Declarations}

\section{Ethical approval and consent to participate}

The study protocol conforms to the 1975 Declaration of Helsinki and was approved by the Institution's human research committee. Written informed consent was obtained from each patient.

\section{Consent for publication}

A written informed consent for publication of results of the present study was obtained from each patient.

\section{Competing interest}

There are no conflicts of interest to declare.

\section{Acknowledgement}

The Authors are grateful to DiaSorin for providing the reagents for iFGF23 testing.

\section{Funding}

No fundings to declare.

\section{Author contribution}

The authors contributed in multiple roles. Giuseppe Vergaro: Conceptualization, Methodology, Visualization. Annamaria Del Franco: Investigation, Visualization, Writing- Original Draft. Francesco Gentile: Investigation, Visualization, Writing- Original Draft. Vincenzo Castiglione: Writing- Original Draft. Alberto Aimo: Writing-Review \& Editing. Federica Saponaro: Investigation. Silvia Masotti: Investigation. Concetta Prontera: Resources, Writing-Review \& Editing. Niccolò Fusari: Data curation, Validation. Michele Emdin: Supervision. Claudio Passino: Supervision.

\section{Availability of data and materials}


The datasets generated and/or analysed during the current study are not publicly available due to the ongoing research for other items of interest in the same enlarged population, but are available from the corresponding author on reasonable request.

\section{References}

1. Silver J, Naveh-Many T. FGF-23 and secondary hyperparathyroidism in chronic kidney disease. Nat Rev Nephrol 9 (2013) 641-9. https://doi.org/10.1038/nrneph.2013.147.

2. Vervloet MG, Massy ZA, Brandenburg VM, Mazzaferro S, Cozzolino M, Urena-Torres P, et al; CKD-MBD Working Group of ERA-EDTA. Bone: a new endocrine organ at the heart of chronic kidney disease and mineral and bone disorders. Lancet Diabetes Endocrinol 2 (2014) 427-36. https://doi.org/10.1016/S2213-8587(14)70059-2.

3. Böckmann I, Lischka J, Richter B, Deppe J, Rahn A, Fischer D, et al. FGF23-Mediated Activation of Local RAAS Promotes Cardiac Hypertrophy and Fibrosis. International Journal of Molecular Sciences. 20 (2019) 4634. https://doi.org/10.3390/ijms20184634.

4. Scialla JJ, Xie H, Rahman M, Anderson AH, Isakova T, Ojo A, et al; Chronic Renal Insuffciency Cohort (CRIC) Study Investigators. Fibroblast growth factor-23 and cardiovascular events in CKD. J Am Soc Nephrol 25 (2014) 349-60. https://doi.org/10.1681/ASN.2013050465.

5. Faul C, Amaral AP, Oskouei B, Hu MC, Sloan A, Isakova T, et al. FGF23 induces left ventricular hypertrophy. J Clin Invest 121 (2011) 4393-408. https://doi.org/10.1172/JCl46122.

6. Olauson H, Vervloet MG, Cozzolino M, Massy ZA, Ureña Torres P, Larsson TE. New insights into the FGF23-Klotho axis. Semin Nephrol 34 (2014) 586-97. https://doi.org/ 10.1016/j.semnephrol.2014.09.005.

7. Akhabue E, Vu TT, Vaidya A, Michos ED, de Boer IH, Kestenbaum B et al. Fibroblast Growth Factor-23, Heart Failure Risk, and Renin Angiotensin-Aldosterone-System Blockade in Hypertension: The MESA Study. Am J Hypertens 32 (2019) 18-25. https://doi.org/ 10.1093/ajh/hpy142.

8. Bergmark BA, Udell JA, Morrow DA, Jarolim P, Kuder JF, Solomon SD, et al. Klotho, fibroblast growth factor-23, and the renin-angiotensin system - an analysis from the PEACE trial. Eur J Heart Fail 21 (2019) 462-70. https://doi.org/10.1002/ejhf.1424.

9. Poelzl G, Trenkler C, Kliebhan J, Wuertinger P, Seger C, Kaser S, et al. FGF23 is associated with disease severity and prognosis in chronic heart failure. Eur J Clin Invest 44 (2014) 1150-8. https://doi.org/10.1111/eci.12349.

10. Ter Maaten JM, Voors AA, Damman K, van der Meer P, Anker SD, Cleland JG, et al. Fibroblast growth factor 23 is related to profiles indicating volume overload, poor therapy optimization and prognosis in patients with new-onset and worsening heart failure. Int J Cardiol 253 (2018) 84-90. https://doi.org/10.1016/j.ijcard.2017.10.010.

11. Gruson D, Lepoutre T, Ketelslegers JM, Cumps J, Ahn SA, Rousseau MF. C-terminal FGF23 is a strong predictor of survival in systolic heart failure. Peptides 37 (2012) 258-62. 
https://doi.org/10.1016/j.peptides.2012.08.003.

12. Stöhr R, Brandenburg VM, Heine GH, Maeder MT, Leibundgut G, Schuh A, et al. Limited role for fibroblast growth factor 23 in assessing prognosis in heart failure patients: data from the TIME-CHF trial. Eur J Heart Fail 22 (2020) 701-9. https://doi.org/10.1002/ejhf.1749.

13. Souberbielle JC, Prié D, Piketty ML, Rothenbuhler A, Delanaye P, Chanson P, Cavalier E. Evaluation of a New Fully Automated Assay for Plasma Intact FGF23. Calcif Tissue Int 101 (2017) 510-8. https://doi.org/10.1007/s00223-017-0307-y.

14. Ponikowski P, Voors AA, Anker SD, Bueno H, Cleland JGF, Coats AJS, et al. 2016 ESC Guidelines for the diagnosis and treatment of acute and chronic heart failure: The Task Force for the diagnosis and treatment of acute and chronic heart failure of the European Society of Cardiology (ESC)Developed with the special contribution of the Heart Failure Association (HFA) of the ESC. Eur Heart J 37 (2016) 2129-200. https://doi.org/10.1093/eurheartj/ehw128.

15. Galderisi M, Cosyns B, Edvardsen T, Cardim N, Delgado V, Di Salvo G, et al. Standardization of adult transthoracic echocardiography reporting in agreement with recent chamber quantification, diastolic function, and heart valve disease recommendations: an expert consensus document of the European Association of Cardiovascular Imaging. Eur Heart J Cardiovasc Imaging 18 (2017) 1301-10. https://doi.org/10.1093/ehjci/jex244.

16. Vergaro G, Aimo A, Prontera C, Ghionzoli N, Arzilli C, Zyw L, et al. Sympathetic and renin-angiotensinaldosterone system activation in heart failure with preserved, mid-range and reduced ejection fraction. Int J Cardiol 296 (2019) 91-7. https://doi.org/ 10.1016/j.ijcard.2019.08.040.

17. Hartman D, Sagnella GA, Chesters CA, Macgregor GA. Direct renin assay and plasma renin activity assay compared. Clin Chem 50 (2004) 2159-61. https://doi.org/ 10.1373/clinchem.2004.033654.

18. Fortunato A, Prontera C, Masotti S, Franzini M, Marchetti C, Giovannini S, et al. State of the art of aldosterone immunoassays. A multicenter collaborative study on the behalf of the Cardiovascular Biomarkers Study Group of the Italian Section of European Society of Ligand Assay (ELAS) and Società Italiana di Biochimica Clinica (SIBIOC). Clin Chim Acta 444 (2015) 106-12. https://doi.org/10.1016/j.cca.2015.01.028.

19. Westermann J, Hubl W, Kaiser N, Salewski L. Simple, rapid and sensitive determination of epinephrine and norepinephrine in urine and plasma by non-competitive enzyme immunoassay, compared with HPLC method. Clin Lab 48 (2002) 61-72. PMID: 11833678.

20. Prontera C, Zucchelli GC, Vittorini S, Storti S, Emdin M, Clerico A. Comparison between analytical performances of polyclonal and monoclonal electrochemiluminescence immunoassays for NTproBNP. Clin Chim Acta 400 (2009) 70-3. https://doi.org/10.1016/j.cca.2008.10.011.

21. Martin A, David V, Quarles LD. Regulation and function of the FGF23/ klotho endocrine pathways. Physiol Rev 92 (2012) 131-55. https://doi.org/10.1152/physrev.00002.2011.

22. Burnett SM, Gunawardene SC, Bringhurst FR, Jüppner H, Lee H, Finkelstein JS. Regulation of Cterminal and intact FGF-23 by dietary phosphate in men and women. J Bone Miner Res 21 (2006) 1187-96. https://doi.org/10.1359/jbmr.060507. 
23. de Borst MH, Vervloet MG, ter Wee PM, Navis G. Cross talk between the renin-angiotensin-aldosterone system and vitamin D-FGF-23- klotho in chronic kidney disease. J Am Soc Nephrol 22 (2011) 16039. https://doi.org/10.1681/ASN.2010121251.

24. Leifeit-Nestler M, Kirchhoff F, Nespor J, Richter B, Soetje B, Klintschar M, et al. Fibroblast growth factor 23 is induced by an activated renin-angiotensin-aldosterone system in cardiac myocytes and promotes the pro-fibrotic crosstalk between cardiac myocytes and fibroblasts. Nephrol Dial Transplant 33 (2018) 1722-34. https://doi.org/10.1093/ndt/gfy006.

25. Hao H, Li X, Li Q, Lin H, Chen Z, Xie J, et al. FGF23 promotes myocardial fibrosis in mice through activation of $\beta$-catenin. Oncotarget 7 (2016) 64649-64. https://doi.org/10.18632/oncotarget.11623.

26. Cilvik SN, Wang JI, Lavine KJ, Uchida K, Castro A, Gierasch CM, et al. Fibroblast growth factor receptor 1 signaling in adult cardiomyocytes increases contractility and results in a hypertrophic cardiomyopathy. PLoS One 8 (2013) e82979. https://doi.org/10.1371/journal.pone.0082979.

27. Grabner A, Schramm K, Silswal N, Hendrix M, Yanucil C, Czaya B, et al. FGF23/FGFR4-mediated left ventricular hypertrophy is reversible. Sci Rep 7 (2017) 1993. https://doi.org/ 10.1038/s41598-01702068-6.

28. Kocełak P, Olszanecka-Glinianowicz M, Chudek J. Fibroblast growth factor 23 - structure, function and role in kidney diseases. Adv Clin Exp Med 21 (2012) 391-401. PMID: 23214203.

29. Block GA, Rosenbaum DP, Yan A, Greasley PJ, Chertow GM, Wolf M. The effects of tenapanor on serum fibroblast growth factor 23 in patients receiving hemodialysis with hyperphosphatemia. Nephrol Dial Transplant 34 (2019) 339-46. https://doi.org/10.1093/ndt/gfy061.

30. Moe SM, Chertow GM, Parfrey PS, Kubo Y, Block GA, Correa-Rotter R, et al. Cinacalcet, Fibroblast Growth Factor-23, and Cardiovascular Disease in Hemodialysis: The Evaluation of Cinacalcet $\mathrm{HCl}$ Therapy to Lower Cardiovascular Events (EVOLVE) Trial. Circulation 132 (2015) 2739. https://doi.org/10.1161/CIRCULATIONAHA.114.013876.

31. Carpenter TO, Whyte MP, Imel EA, Boot AM, Högler W, Linglart A, et al. Burosumab Therapy in Children with X-Linked Hypophosphatemia. N Engl J Med 378 (2018) 1987-98. https://doi.org/ 10.1056/NEJMoa1714641.

\section{Figures}

\section{Figure 1}

Baseline characteristics of patients in each FGF23 tertile. Cardiorespiratory performance, laboratory examinations and echocardiography parameters of patients in the first $(T 1,<35.2 \mathrm{pg} / \mathrm{mL})$, second (T2, $35.2-50.9 \mathrm{pg} / \mathrm{mL})$ and third $(\mathrm{T} 3,>50.9 \mathrm{pg} / \mathrm{mL})$ iFGF23 tertile. 
${ }^{*} P<0.05$ vs. first tertile, $+P<0.05$ vs. second tertile. eGFR, estimated Glomerular Filtration Rate; LVEF, left ventricular ejection fraction; LVMI, left ventricular mass index; NT-proBNP, N-terminal pro B-type natriuretic peptide; PRA, plasma renin activity; PTH, parathormone.

\section{Figure 2}

FGF23 tertiles and outcome. Kaplan-Meier curves depicting rates of 5-year all-cause mortality (a), cardiac mortality (b) and of the composite of all-cause mortality and heart failure hospitalizations (c) according to iFGF23 tertiles.

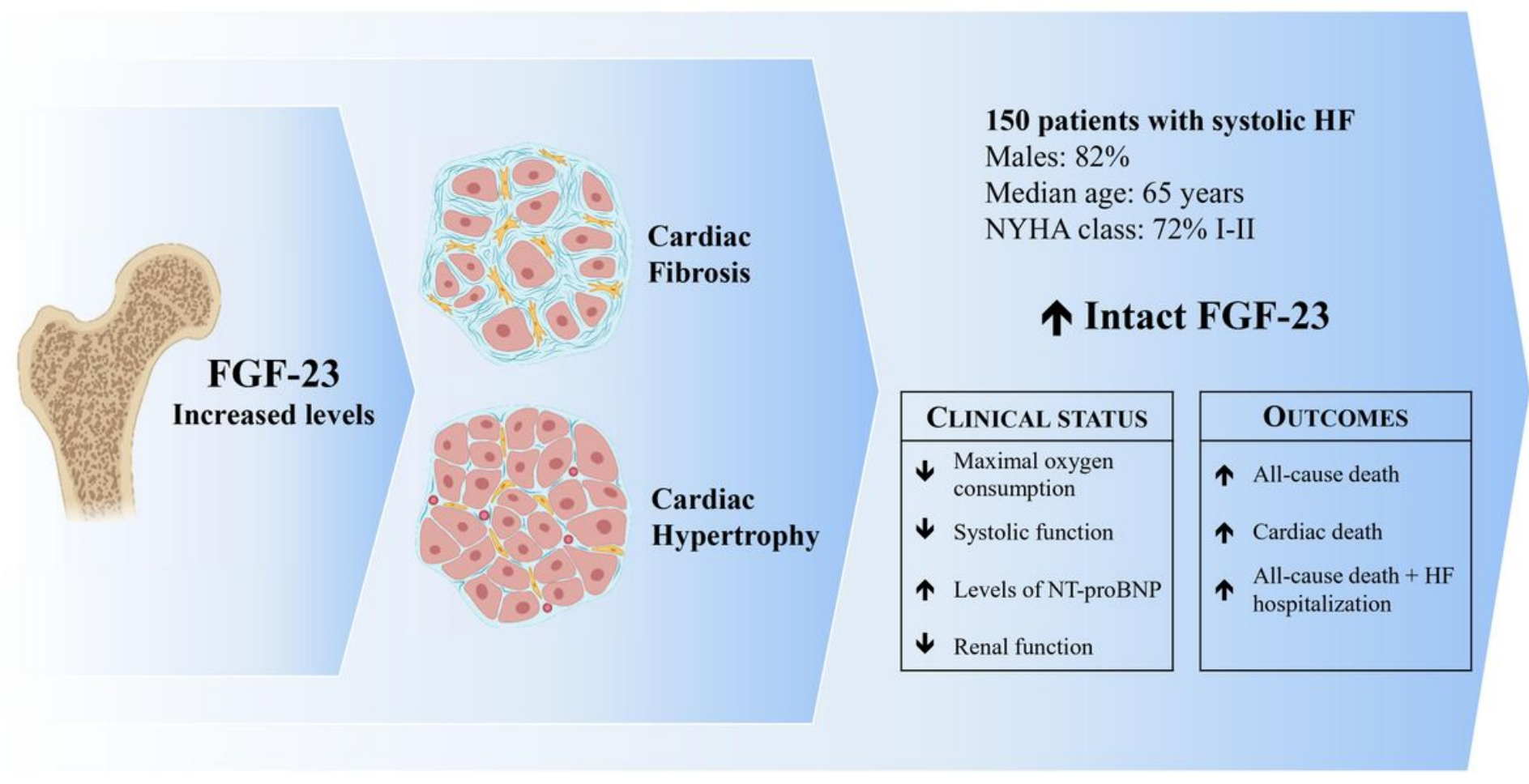

Figure 3

Prognostic impact of FGF23 levels in patients with systolic heart failure (HF). In a cohort of HF patients with systolic dysfunction, higher iFGF23 levels (index of increased cardiac fibrosis and hypertrophy) were associated with clinical severity of the disease, as expressed by lower left ventricular systolic function, higher circulating levels of $\mathrm{N}$ - terminal pro-brain natriuretic peptide (NT-proBNP), lower oxygen consumption at cardiopulmonary exercise test and worsening of renal function. Moreover, iFGF23 elevation identified patients at higher risk for all-cause and cardiac mortality, and HF hospitalization. 